\title{
Synthesis and Characterization of ZnO Nanostructures with Macroscopic Gel- like Morphology
}

Dinghao Tang, Hongyang Liu and Jingyue (Jimmy) Liu

Center for Nanoscience and Department of Physics and Astronomy, University of Missouri-St. Louis, One University Boulevard, St. Louis, Missouri 63121, USA (liuj@umsl.edu)

As an important II-VI semiconductor material, zinc oxide $(\mathrm{ZnO})$ and its nanostructures have attracted great attention in the last decade because of its potential applications in various fields, such as sensing, photocatalysis, field emission, and disease diagnostics and treatment [1]. Due to nonsymmetric wurtzite structure, $\mathrm{ZnO}$ nanostructures possess various morphologies such as nanowires, nanobelts, nanoring, nanohelixes, nanotetrapods, etc., which have been synthesized by using various physical or chemical methods [1]. Most macroscopic morphologies of $\mathrm{ZnO}$ nanomaterials, synthesized by physical vapor deposition method, however, look like white-wool morphology or the variations of it. We report here the synthesis of gel-like $\mathrm{ZnO}$ films.

The $\mathrm{ZnO}$ nanostructures were synthesized in a high temperature tube furnace by a standard thermal evaporation-condensation process. The experimental setup was similar to those reported in literature [2]. We used a mixture of oxygen and argon as the carrier gas. The morphology of as-grown nanostructures was examined in a high-resolution field emission SEM (JEOL JEM-6320F).

Figure 1 is an optical digital image of as-grown $\mathrm{ZnO}$ white gel-like materials. The gel-film is very soft and lightweight and can be easily torn. These structures sometimes look like a film and sometimes look like a paste. They usually grew in the lower temperature zone of the tube furnace with temperatures ranging from about $400^{\circ} \mathrm{C}$ to $500^{\circ} \mathrm{C}$. Large-scale synthesis of such structures is possible. Figure 2 shows a SEM image of the as-grown gel, revealing the presence of nanowires and some multipods. Detailed examination of many SEM images showed that almost all the nanowires are connected with each other; some multipods were also observed as shown in the image. Figure 3 is a high magnification SEM image of a typical nanowire showing the morphology of the nanowire and the regularity of the change of its diameter. Detailed measurements of the diameters of the nanowires showed that there was a bimodal distribution of nanowire diameters with their average sizes of $53 \mathrm{~nm}$ and $125 \mathrm{~nm}$, respectively. The clusters usually connect the nearby nanowires together tightly, which is central for the macroscopic gel-like morphology. The pressure during the growth of as-grown nanomaterials is also a key factor. The growth mechanism of gel-like morphology as well as the detailed synthesis-structure relationships will be discussed [3].

\section{References}

[1] Z.L. Wang, Mate. Sci. \& Eng. R. 64 (2009) 33.

[2] Z. W. Pan et al., Science 291 (2001) 1947.

[3] This research was supported by the University of Missouri-St. Louis. 


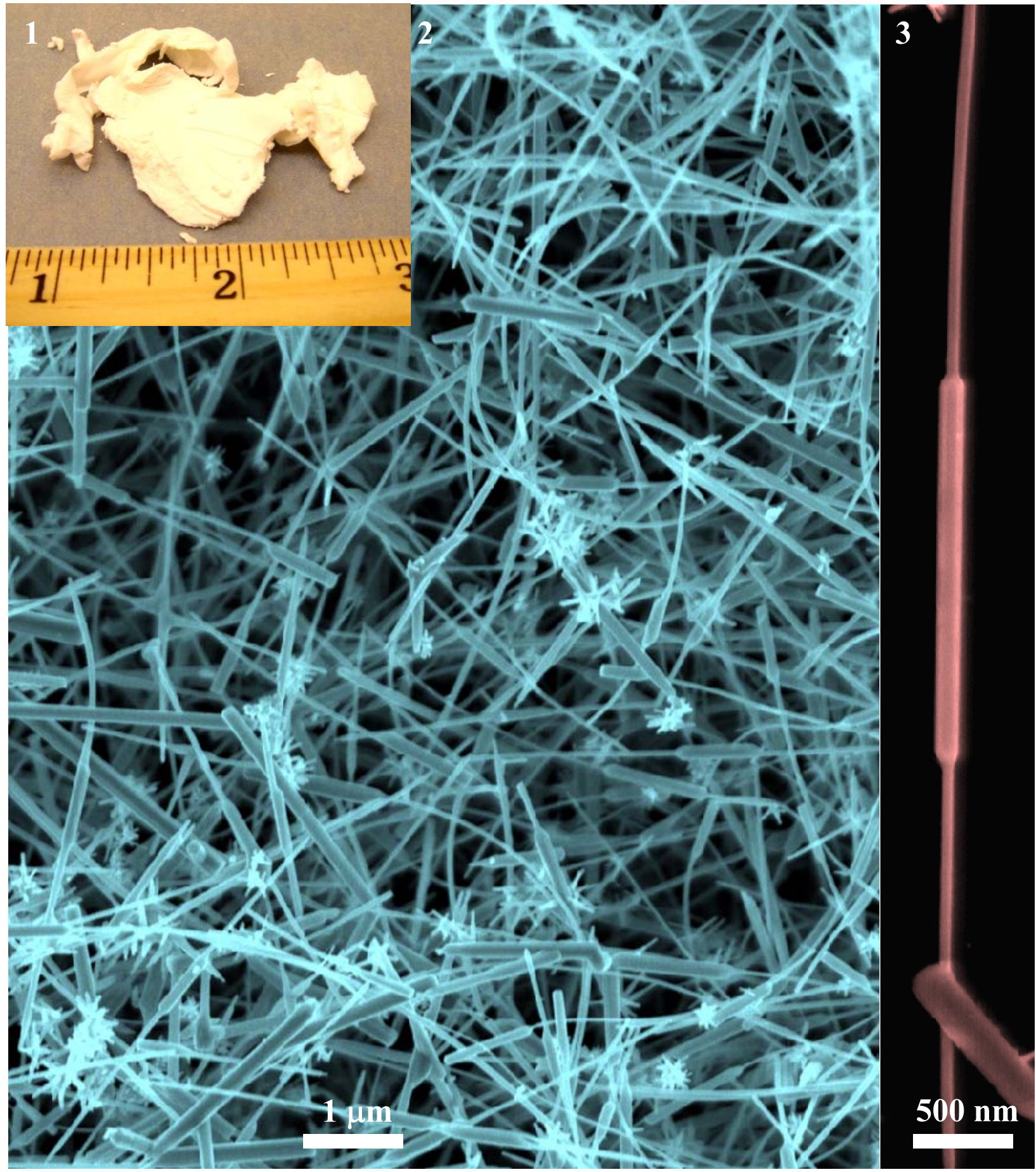

FIG. 1. Optical digital image of as-grown nanomaterials showing the macroscopic soft gel-like morphology.

FIG. 2. SEM image of as-grown $\mathrm{ZnO}$ soft gel revealing the interlacing and networked $\mathrm{ZnO}$ nanowires as well as some nanomultipods.

FIG. 3. SEM image of a typical nanowire within the $\mathrm{ZnO}$ gel-like structure showing the bimodal distribution of the nanowire diameters and the joint of different nanowires. 